\title{
The Effectiveness of Hyperbaric Oxygen Therapy in an Established Intracerebral Tumor Model
}

\author{
Tolga Turan DÜNDAR¹, Mehmet Hakan SEYITHANOĞLU'1, Şefika KORPINAR², Raziye ATAR ${ }^{3}$, Şevket EVRAN ${ }^{1}$ \\ ${ }^{1}$ Department of Brain and Nerve Surgery, Bezmialem Vakif University Faculty of Medicine, Istanbul, Turkey \\ ${ }^{2}$ Private Medok Hyperbaric Oxygen Therapy Centre, Istanbul, Turkey \\ ${ }^{3}$ Institute of Cardiology, Istanbul University, Istanbul, Turkey
}

\begin{abstract}
Objective: Gliomas are the most common brain tumor in adults. Tumor-related endothelial cells are of neoplastic nature and have malignant properties such as proliferation and invasion. Therefore, antiangiogenic approaches have several advantages compared with conventional chemotherapeutic approaches. The target of antiangiogenic treatment is to ensure a decrease in systemic side effects because there are endothelial cells and other supportive cells belonging to the vessel walls. The purpose of this study was to increase the oxygenation of the environment with hyperbaric oxygen therapy (HBOT) and to prevent the angiogenic effect of hypoxia-related factors. We also tried to prevent angiogenesis by using sorafenib (SOR), which is a chemotherapeutic agent that blocks the receptors of these factors. Also, this study compared the effect of these both and to put into for their effect. In this regard, parameters such as the tumor size, vascularization rate, midkine (MK), apoptosis- and angiogenesis-related transcription tumor necrosis factor (TNF) alfa, and IL6 were compared in the relevant groups.

Methods: For this purpose, a convenient trial model was formed and the following 4 groups were determined: The study population was divided into four main groups: G1-control group, G2-group given HBOT, G3-group given SOR, G4-group given HBOT and SOR. Ten rats were included in each group. After 2 weeks, the rats were sacrificed. After being sacrificed based on the date and surgery determined, the tumor volume and vascularization ratio were checked with light microscope. Serum samples are evaluated by using ELISA for TNFalpha, Interleukin-6 (IL-6) and midkine (MK) levels.

Results: The underlying mechanism of HBOT by evaluating MK levels was shown for the first time in this study. We have found that HBOT potentializes the sorafenib efficacy. HBOT increased MK levels and HBOT decreased anti-apoptotic TNF- $\alpha$ and IL- 6 levels. MK levels show inverse correlation with TNF- $\alpha$ levels and IL-6 levels. TNF- $\alpha$ and IL-6 levels are directly proportional.
\end{abstract}

Conclusion: There is a need for further studies to evaluate the correct time of application of HBOT.

Keywords: HBOT, sorafenib, GBM, IL6

\section{Introduction}

Gliomas are the most common brain tumors seen in adults. They constitute approximately one-third of primary brain tumors. According to genetic and cytological findings, high-grade gliomas have a significant heterogeneous etiopathogenesis. The differences in survival rates and responses to treatment among patients are because of this genetic etiological variation (1). Despite the current advanced examination and treatment opportunities, the 5-year survival rate is lower than $5 \%$ for glioblastoma multiforme, which is the most common and the most aggressive type among these tumors (2-4).

Hypoxia plays an important role in embryogenesis and in the growth of cancer tissues. Hypoxia causes the secretion of many growth factors, leading to vascularization and increased tumor size. Vascular endothelial growth factor (VEGF) and platelet-derived growth factor (PDGF) are only two of these secreted factors. Hypoxia increases the levels of these factors. The chemotherapeutic agent called sorafenib is the receptor blocker of these two factors. In this study, it was aimed to prevent the effect of factors secreted because of hypoxia on tumor angiogenesis by increasing the oxygenation of the environment with hyperbaric oxygen therapy (HBOT).

This study was presented as oral presentation at the $2^{\text {nd }}$ International Excellence in Midkine Research Conference, 28-30 June 2012, Istanbul, Turkey. Address for Correspondence: Tolga Turan Dündar; Department of Brain and Nerve Surgery, Bezmialem Vakif University Faculty of Medicine, i̇stanbul, Turkey. Phone: +9021233192 29 E-mail: drtolgadundar@hotmail.com 
Sorafenib is an orally used receptor tyrosine kinase inhibitor that decreases the proliferation of tumor cells. It was aimed to prevent angiogenesis using sorafenib that blocks VEGF and PDGF receptors in this study.

Furthermore, comparing both factors (hyperoxia-sorafenib) and revealing their efficiency together were the goals of our study. In this regard, parameters such as tumor size, vascularization rate, midkine (MK), apoptosis- and angiogenesisrelated tumor necrosis factor (TNF) alpha, and interleukin-6 (IL-6) were compared in the relevant groups.

\section{Methods}

\section{Experimental Design}

This study was conducted with the support of Bezmialem Foundation University, Faculty of Medicine BAP. It was approved by the Ethics Committee of Istanbul University, Institute of Experimental Medicine Research (Protocol no: 2011/03). In the study, 9-month inbred Wistar albino male rats (weight 200-300 g) were used. The rats were kept in light conditions for $14 \mathrm{~h}$ and in dark conditions for $10 \mathrm{~h}$, and they were fed with commercial rat food.

Four main groups were formed for the study: Group 1 (control group): The group in which the tumoral tissue was left in its natural course following tumor implantation after a surgical process was applied. Group 2 (the group given HBOT): Surgical intervention was performed as explained and tumor implantation was conducted. On the $7^{\text {th }}$ day, tumor implantation was confirmed with cranial magnetic resonance imaging (MRI). After the $7^{\text {th }}$ day, HBOT was initiated at a pressure of 2.5 ATA for 90 min twice a day. Group 3 (the group given sorafenib): After tumor implantation was performed, tumor implantation was confirmed with cranial MRI on the $7^{\text {th }}$ day. On the $7^{\text {th }}$ day, a $10 \mathrm{mg} /$ $\mathrm{kg}$ dose of sorafenib was administered orally. Group 4 (the group given HBOT and sorafenib): After tumor implantation, tumor implantation was confirmed with cranial MRI on the $7^{\text {th }}$ day. On the $7^{\text {th }}$ day, sorafenib at a dose of $10 \mathrm{mg} /$ $\mathrm{kg}$ per day was initiated with HBOT. For 14 days, a single dose of sorafenib was given in a day, and HBOT was applied at a pressure of 2.5 ATA for 90 min twice a day.

The surgical procedure was performed at Istanbul University, Institute of Experimental Medicine Research. In total, 106 C6 rat glial cells were implanted to the right frontal lobes of the rats with a cranial anterior-parasagittal burrhole (Picture 1). The rat C6 glioma cell line (CCL-107) used in the study was obtained from the cell bank in the American Type Culture Collection (ATCC) and produced in the Cell and Tissue Culture Laboratory of Istanbul University Medicine Faculty. On the $7^{\text {th }}$ day after implantation, cell proliferation was confirmed with cranial MRI. The signal change in the right frontal lobe was accepted to be positive and groups were formed. HBOT was applied in the cabin of
Oxygen at Istanbul University. On the $21^{\text {st }}$ day, decapitation was performed and serum samples were collected (Picture 1).

TNF alpha, IL-6, and MK levels were evaluated with enzymelinked immunosorbent assay (ELISA). Necrosis and vascularization rates were determined with hemotoxylin-eosin staining. After the area of tumor was calculated in $\mathrm{mm}^{2}$, it was multiplied by section thickness for identifying the volume of the tumor. The intratumor vascularization rate was determined as a $\%$ value with the following formula: intratumor vessel number/total tumor area $\times 100$. The Statistical Package for the Social Sciences (SPSS) 17.0 software was used for statistical analysis. Statistical analyses of all data were performed using Student's ttest according to variance analyses, and the results were demonstrated as mean \pm standard deviation (SD). The values of $\mathrm{p}<0.05$ were accepted to be statistically significant.

\section{Results}

Detection of Tumor Volume and Vascularization Rate with an Optical Microscope: It was found that all applications decreased tumor volume according to the control group $(38 \pm 8.3$ $\mathrm{mm}^{3}$ ). With respect to SOR and HBOT application, the highest decrease in tumor volume was detected in the combination group (PSOR <0.01, PHBOT <0.001) (Figure 1).

Intratumor vascularization was found to be $6.1 \pm 0.7 \%$ in the control group. It was detected that this vascularization rate decreased in all groups. The highest decrease was in the combination group $(3.78 \pm 0.1 \%$; PCONTROL $<0.001$; PHBOT $<0.01$; PSOR $<0.01)$ and then in the SOR group $(4.24 \pm 0.9 \%$; PCON-

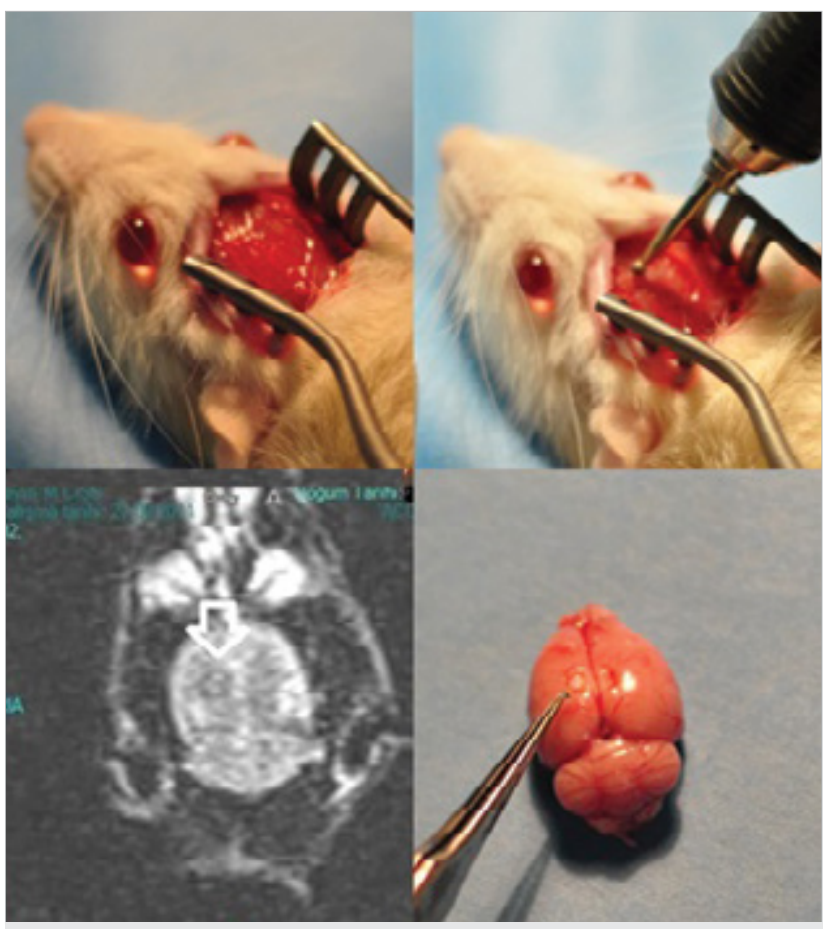

Picture 1. The sequential images of the surgical process and confirmation through cranial MRI of the rat. After all, the demonstration of the tumoral structure in sacrificed brain tissue 
TROL < 0.05 ; PHBOT <0.01; PCOMBINATION <0.01) and in the HBOT group $(4.9 \pm 0.2 \%$; PCONTROL $<0.05$; PSOR $<0.01$; PCOMBINATION <0.01) (Figure 2, Picture 2).

Detection of TNF Alpha and IL6 Levels with ELISA: It was found that HBOT decreased TNF alpha levels $(p<0.01)$ and sorafenib increased TNF alpha levels $(\mathrm{p}<0.05)$; these levels were similar in the combination and control groups $(\mathrm{p}>0.05)$ (Figure 3 ).

Moreover, it was also revealed that HBOT caused a decrease in IL-6 levels $(\mathrm{p}<0.01)$, and sorafenib increased IL-6 levels $(\mathrm{p}<0.05)$; these values were similar in the control and combination groups (Figure 4).

Detection of MK Levels with ELISA: HBOT increased the levels of MK at the highest rate $(\mathrm{p}<0.01)$ and sorafenib followed it $(\mathrm{p}<0.01)$. Despite this, the values in the combination aaroups were similar $(\mathrm{p}>0.05)$ (Figure 5).

\section{Discussion}

Hypoxia is the main driving force for neovascularization. This phenomenon, which contributes to the tissue development in the fetal period, also contributes to the development of a tumor in the tumoral tissue, and it causes a decrease in the response of the tumor tissue to treatment.

The proteins located in the membrane are called receptor tyrosine kinase (RTK). Fifty-eight transmembrane proteins are found in the RTK super family. Among these receptors, there

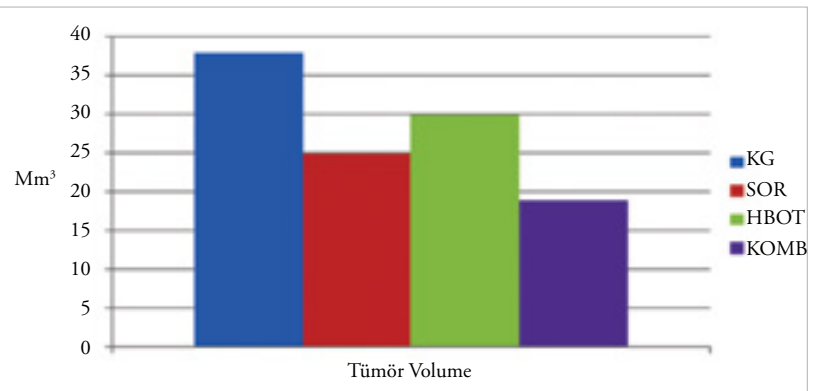

Figure 1. The images were obtained after hemotoxylin-eosin staining, and they were evaluated using NIH Image software. After the area of tumor was calculated in $\mathrm{mm}^{2}$, it was multiplied by section thickness for identifying the volume of the tumor

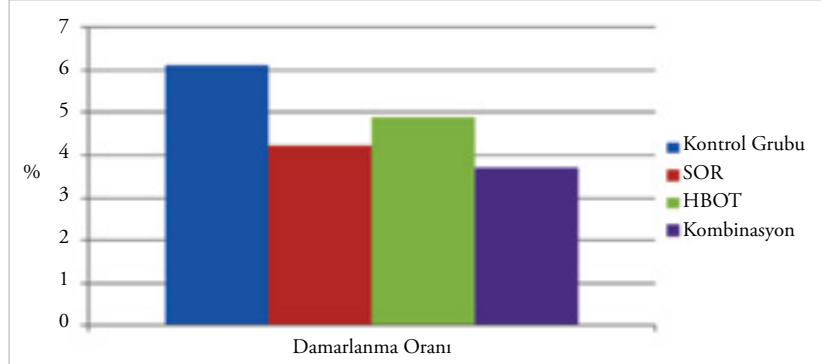

Figure 2. Intratumor vascularization rate was determined as a \% value with the following formula: intratumor vessel number/total tumor area $\times 100$ are insulin receptors, growth factors (EGF, VEGF, PDGF, FGF, NGF) receptors, and ephrin receptors (EphA, EphB) (7). Continuous and uncontrolled RTK activity is the case during the carcinogenesis process (8). The chemotherapeutic drug named sorafenib is the new generation antineoplastic agent that prevents neovascularization.

MK is a protein-binding carbohydrate and defined as a cytokine or growth factor. It has a role in growth, migration, and gene expression in various target cells. Reproduction plays a pathological role in many diseases besides its repair duty. The prognosis of the patients with MK levels at a high rate were found to be poor compared with the patients having low levels of MK $(9,10)$. TNF- $\alpha$ and IL- 6 are the moderators revealed in both cell transformation and neovascularization (11).

\section{Conclusion}

In this study, the efficiency of HBOT therapy and sorafenib, which is a well-known and commonly used antineoplastic

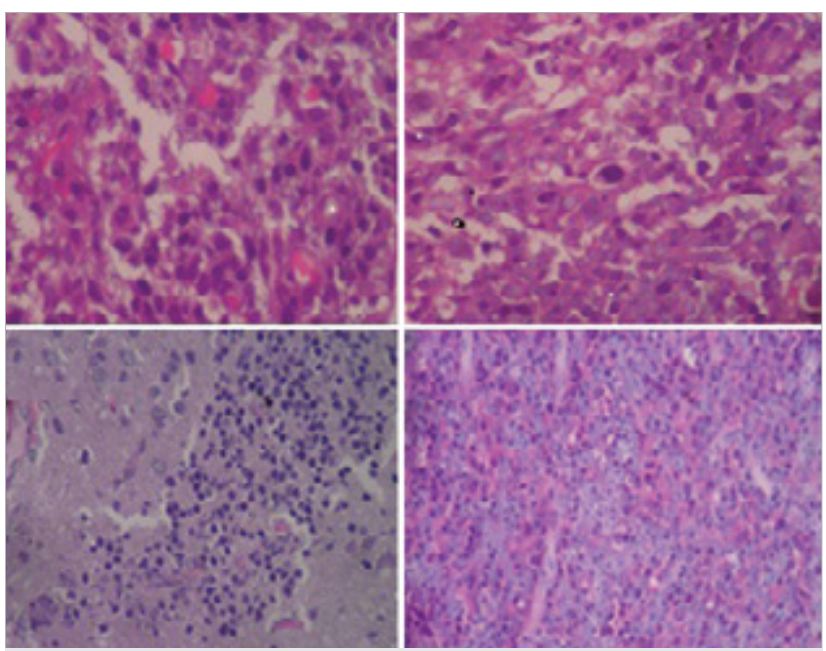

Picture 2. Demonstration of images obtained after hemotoxylin-eosin staining with a camera (Diagnostic Instruments, Inc., Sterling Heights, MI)

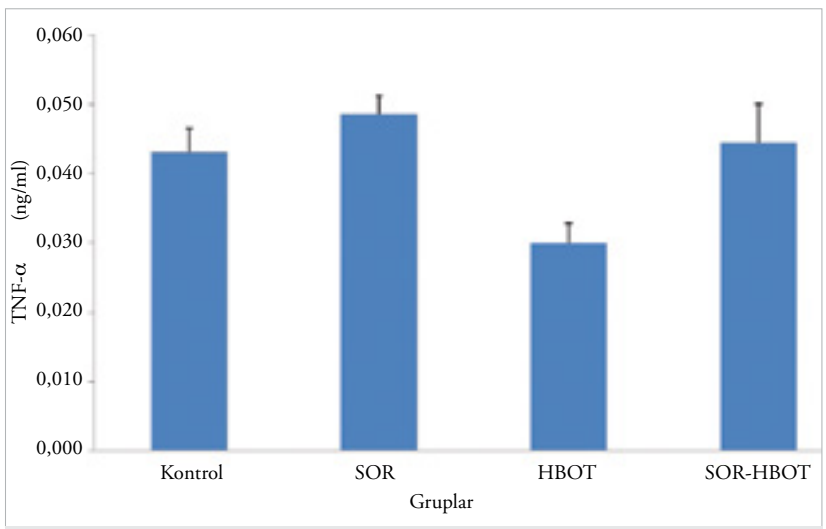

Figure 3. Serum TNF alpha levels $(\mathrm{ng} / \mathrm{mL})$ with ELISA. It is observed that SOR and combination groups increased TNF alpha levels compared with the control group. HBOT group decreased TNF alpha levels compared with the control group 


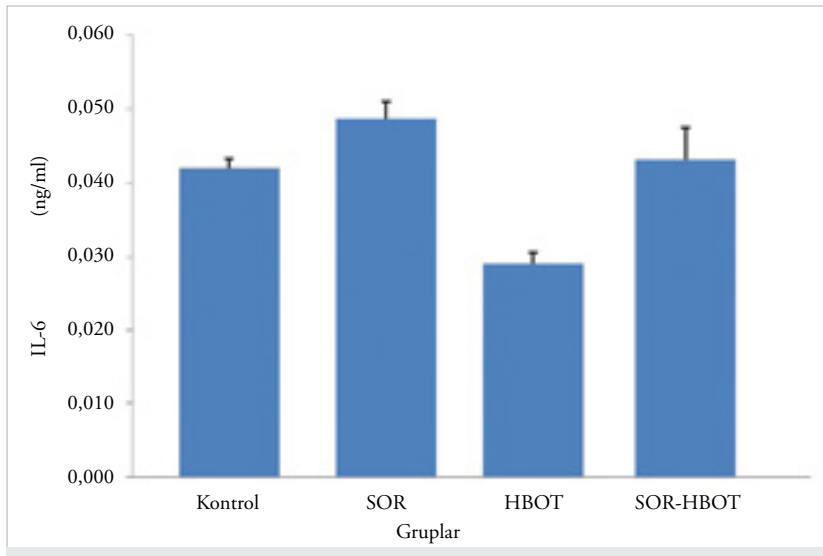

Figure 4. Serum IL-6 levels with ELISA (ng/mL). The change in IL-6 levels was found to be similar to the change in TNF alpha levels

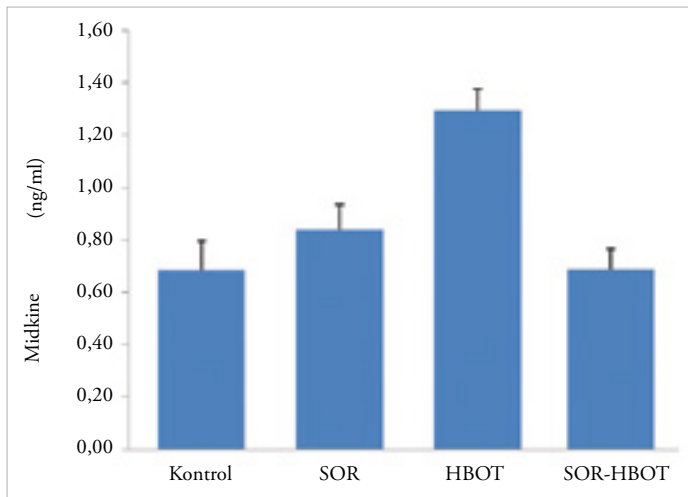

Figure 5. Serum midkine levels with ELISA (ng/mL). It was found that the HBOT group increased midkine levels and the combination group decreased the levels compared with the control group

agent, combination therapy was investigated. It was detected for the first time that HBOT displayed a synergistic effect with sorafenib, it increased serum MK levels, and it decreased anti-apoptotic TNF- $\alpha$ and IL- 6 levels. Although sorafenib decreases MK levels in human T98G GBM cell cultures, it was found to increase these levels in this in vivo study. Furthermore, it was detected that HBOT decreased the cytotoxicity of sorafenib and potentialized its effect on the tumoral tissue. As our knowledge on the molecular level increases, it is seen that responsible molecules can display opposite effects in some situations. This shows that a delicate balance is under the control of many mechanisms, even in pathological process. Multi-center studies are needed for understanding these mechanisms better.

Ethics Committee Approval: Ethics committee approval was obtained.

Peer-review: Externally peer-reviewed.

Author Contributions: Concept - T.T.D.; Design - T.T.D., M.H.S.; Supervision - M.H.S.; Funding - T.T.D.; Materials - Ş.K., R.A.; Data Collection and/or Processing - T.T.D.; Analysis and/or Interpretation - T.D.; Literature Review - T.T.D., R.A., Ş.K.; Writer - T.T.D.; Critical Review - M.H.S., Ş.K.

Conflict of Interest: The authors declared no conflict of interest.

Financial Disclosure: The authors supported by the Bezmialem Vakıf University BAP Support.

\section{References}

1. World Health Organization, International agency for research on cancer. WHO Classification of Tumours of the Central Nervous System. Fourth edition, International Agency for Research on Cancer, Lyon, 2007.

2. Ohgaki H, Dessen P, Jourde B, Horstmann S, Nishikawa T, Di Patre PL, et al. Genetic pathways to glioblastoma: a population-based study. Cancer Res 2004; 64: 6892-9. [CrossRef]

3. CBTRUS Statistical Report: Primary Brain and Central Nervous System Tumors Diagnosed in the United States in 2004-2008. CBTRUS 2012; 1-48.

4. Eisele G, Weller M. Targeting apoptosis pathways in glioblastoma. Cancer Lett 2011;335-45.

5. Folkman J. What is the evidence that tumors are angiogenesis dependent? J Natl Cancer Inst 1990; 82: 4-6. [CrossRef]

6. Ferrara N. VEGF: an update on biological and therapeutic aspects. Curr Opin Biotechnol 2000; 11: 617-24 . [CrossRef]

7. Blume-Jensen P, Hunter T. Oncogenic kinase signaling. Nature 2011; 411: 355-64. [CrossRef]

8. Doğan L, Güç D. Sinyal iletimi mekanizmaları ve kanser. Hacettepe Tip Dergisi 2004; 35: 34-42.

9. Muramatsu T. Midkine, a heparin-binding cytokine with multiple roles in development, repair and diseases. Jpn Acad Ser B Phys Biol Sci 2010; 86: 410-25. [CrossRef]

10. Mishima, K, Asai A, Kadomatsu K, Ino Y, Nomura K, Narita Y, et al. Increased expression of midkine during the progression of human astrocytomas. Neurosci Lett 1997; 233: 29-32. [CrossRef]

11. Suganuma M, Okabe S, Kurusu M, Iida N, Ohshima S, Saeki Y, et al. Discrete roles of cytokines, TNF-alpha, IL-1, IL-6 in tumor promotion and cell transformation. Int J Oncol 2002; 20: 131-6. 